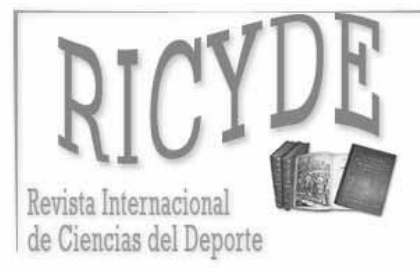

International J ournal of Sport Science VOLUMEN II. AÑO II

Páginas:20-43 ISSN:1885-3137

№ 3 - abril - 2006

\title{
Actitudes hacia la práctica físico-deportiva según el sexo del practicante \\ Gender and attitudes toward the practice of physical activity and sport
}

\author{
J uan Antonio Moreno Murcia \\ Universidad de Murcia \\ Celestina Martínez Galindo y Néstor Alonso Villodre \\ Unidad de Investigación en Educación Física y Deportes
}

\section{Resumen}

El objetivo principal del estudio es examinar las actitudes hacia la práctica físico-deportiva según el sexo del practicante, con el fin de conocer y comprender los diferentes motivos que conducen a practicar actividades físicodeportivas. Para ello, se analizan las diferentes valoraciones otorgadas por el alumnado a la Educación Física, las prácticas físico-deportivas atendiendo a las diferencias existentes por sexo, la importancia de la motivación y la diversión en la práctica de actividad física regular, la aceptación social de las mujeres en el deporte y también, basándonos en todo lo expuesto, se establecen algunas prospectivas de estudio.
Los resultados de los estudios muestran que las preferencias de los varones hacia la práctica físico-deportiva corresponden a actividades colectivas y competitivas, tales como el fútbol y el baloncesto, mientras que las adolescentes y mujeres muestran, tanto en la etapa educativa como post-educacional, actitudes positivas hacia actividades de tipo individual y estéticas tales como el aeróbic y la natación, actividades no ofertadas, en la mayoría de los casos, en las clases de Educación Física. Este aspecto ha sido considerado por los expertos como fomentador de desigualdad de oportunidades así como de discriminación del sexo femenino.

Palabras clave: género, igualdad de trato, coeducación, educación física, deporte. 


\title{
Gender and attitudes toward the practice of physical activity and sport
}

\section{Actitudes hacia la práctica físico-deportiva según el sexo del practicante}

\author{
J uan Antonio Moreno Murcia \\ Universidad de Murcia \\ Celestina Martínez Galindo y Néstor Alonso Villodre \\ Unidad de Investigación en Educación Física y Deportes
}

\begin{abstract}
The principle objetive of the study is to examine the attitudes toward the practice of sports according to the sex of the practitioner, with the final objetive being to understand and describe the different motivations that drive them to exercise. For this, analysis is given to the different valuations given by the student body toward physical education. These include various physical activities practiced according to gender, the importance of motivation and "fun factor" in the practice of regular exercise, social acceptance of women in sport, and also, based on the results obtained, to establish various conclusions cocerning the study.

The results demostrate that the preferences of males toward exercise tend to correspond with group activities and those that are competitive, such as soccer and basketball, whereas adolescents and women demonstrate that in the educational stage as well as post educational, that they have more positive attitudes toward individual activities such as aerobics and swimming, activities normally not offered in the majoriry of case in physical education classes. Many experts consider these factors as things that help promote opportunities as well as female sex descrimination.
\end{abstract}

Key Words: sex, equality of treatment, co-education, Physical Education, sport.

Correspondence: Dr. Juan Antonio Moreno Murcia, e-mail: morenomu@um.es 


\section{Introducción}

Un amplio número de investigaciones han confirmado los indudables beneficios físicos y psicológicos que aporta la actividad física practicada de forma regular, produciendo un impacto importante en el bienestar y en la calidad de vida de quienes la practican. En línea con estas afirmaciones, la evidencia sería un incremento paulatino de participación en actividades físico-deportivas, sin embargo, contrario a esta afirmación lógica, existe un tendente declive en el disfrute de tales actividades a lo largo de la vida (Owen y Bauman, 1992).

Recientes estudios indican que más del $60 \%$ de los sujetos adultos no se compromete en actividades físico-deportivas (Jones, Ainsworth, Croft, Macera, Lloyd y Yusuf, 1998) y que el $25 \%$ de la población adulta resulta completamente sedentaria. Además, las investigaciones han determinado que aproximadamente el $50 \%$ de los sujetos que comienzan una actividad física abandona su práctica entre los primeros 3 y 6 meses (Dishman, 1988). Del mismo modo, y centrándonos en la variable referente al género, diferentes investigaciones han mostrado que la chicas adolescentes participan menos en las clases de Educación Física (Chepyator-Thomson y Ennis, 1997), así como que existe un incremento del número de chicas universitarias que abandonan la participación en actividades físicas-deportivas (Douthitt, 1994 y Jaffee y Richer, 1993).

En este sentido, la severidad y permanencia de estilos de vida sedentarios, el marcado descenso de participación y la carencia de adherencia a programas físicos-deportivos, fundamentalmente entre las chicas, ha motivado que nos preocupemos por estudiar las actitudes hacia la práctica físico-deportiva según el sexo del practicante, con el fin de conocer y comprender los diferentes motivos que nos mostrarán algunas características para poder diseñar programas de actividades físico-deportivas con la intención de poder aumentar la adherencia a programas de estas características durante la etapa adulta, centrándonos en los adolescentes, y más concretamente, en el género femenino.

\section{Estereotipos y actitudes sexistas en Educación Física}

En el seno de cada sociedad se van configurando estereotipos asociados al género, la edad, así como a cualquier otro parámetro como la raza, nacionalidad, etc., en definitiva, se van interiorizando una serie de formas de sentir, pensar y actuar que determinan papeles sociales diferentes según el sexo (Sicilia, 2002) y sirven como referencia para la atribución de un determinado estatus, la expectativa del cumplimiento de un rol consecuente con el anterior, así como el interés por practicar un determinado deporte o práctica físico-deportiva. Por lo tanto, la fuerza de estos estereotipos físicosociales va a repercutir en el hecho de que los alumnos lleguen a la escuela con unas actitudes sexistas, reforzadas por la intervención educativa, llevando todo ello paulatinamente a las adolescentes hacia una desmotivación por la Educación Física y, consecuentemente, hacia una serie de consecuencias, tales como una escasa 
participación en actividades físico-deportivas extraescolares, una falta de adherencia en programas de estas características durante la etapa post-educativa y un crecimiento de los índices de abandono durante la edad adulta.

Algunas de estas actitudes sexistas adquiridas por los alumnos hacen referencia al hecho de que la mayoría de niñas y adolescentes, sobre todo a partir de los 13-14 años, rechazan los esfuerzos de cierta intensidad (trabajo de resistencia, potencia, etc.), mientras que los niños, por el contrario, suelen aceptar mejor este tipo de esfuerzos, bien sea por la imitación de determinados deportistas ó bien por la imagen social y familiar implícita en el deporte. Del mismo modo, la mujer adolescente, en general, observa una mayor identificación de la actividad deportiva con el varón que con ella misma, aunque aceptan participar en actividades masculinas en mayor medida que los chicos lo hacen con actividades catalogadas como femeninas, tal es el caso de la expresión corporal o la danza. Otra actitud sexista hace mención al hecho de que la propia mujer se sitúe en condiciones de inferioridad en cuanto a sus posibilidades de aprendizaje, lo que determina la falta de motivación femenina hacia la actividad física. Debido a esta falta de motivación, así como por el complejo de inferioridad, la mujer evita entrar en competencia con el sexo opuesto en el campo de la actividad física, un sexo seguro de sus posibilidades y con liderazgo. Otro de los motivos que puede condicionar la interacción entre alumnos en el desarrollo de actividades físicas es el nivel de "agresividad física" a través de contactos corporales reglados que suelen ser mucho más utilizados por los chicos que por las chicas. Finalmente, la menstruación, los efectos negativos sobre el aparato reproductor femenino y la masculinización de la mujer, han sido los grandes temas sobre los que se ha asentado la inhibición deportiva de la mujer y, aunque actualmente dichos prejuicios parecen carecer de sentido, las adolescentes suelen emplearlo como "excusa” para rechazar la realización de actividad físico-deportiva.

En definitiva, para comprender la construcción ideológica que determina los intereses y actitudes hacia la actividad físico-deportiva debemos tener en consideración diversos aspectos que abarcan desde aspectos que abarcan desde la valoración realizada por los discentes sobre la Educación Física, el interés de la población por practicar determinadas actividades físico-deportivas, la importancia de aspectos tales como la motivación y la diversión en la adopción de estilos de vida activos y la aceptación social de la mujer en el mundo del deporte, atendiendo, para ello, a las diferencias de sexo existentes. Aspectos que abarcaremos en el siguiente estudio.

\section{Valoración de la Educación Física según el sexo}

En los últimos años ha habido un incremento de estudios centrados en el análisis de las actitudes de los alumnos hacia la Educación Física. Este incremento puede ser atribuido a la influencia que las actitudes ejercen sobre la participación futura en actividades físicas fuera de la etapa escolar (Carlson, 1995; Ennis, 1996; Portman, 1995), sobre el éxito de los alumnos en la Educación Física (Graham, 1995; Lee, 1997) y sobre el conocimiento otorgado a través de las actitudes de los alumnos hacia la Educación Física en vistas al desarrollo del currículum (Cothran y Ennis, 1998). Las vivencias específicas que suponen las clases de Educación Física dan lugar a una valoración de las 
mismas por parte del alumnado. Esta valoración, en conjunto, conformará actitudes positivas ó negativas que, a su vez, generarán hábitos de práctica de ejercicio físico, o por el contrario, desligarán a la persona de toda práctica físico-deportiva a lo largo de su vida adulta (Moreno y Cervelló, 2003).

Recientemente ha habido una creciente concienciación acerca de las marcadas actitudes negativas que muestran las adolescentes femeninas hacia la actividad física en general y la Educación Física en particular. Esta pronunciada negatividad toma algunas formas y ha sido bien documentada por autores tales como Scraton (1992) y Shropshire, Carroll y Yim (1997), según los cuales estas formas oscilan entre el escaso entusiasmo hacia las actividades deportivas extra-curriculares, la no participación en las mismas y la ausencia de clases de Educación Física. Por su parte, Deem y Gilroy (1998), señalan como principales razones que explican las actitudes negativas mostradas por las alumnas, su propia habilidad atlética, la aceptación social de las chicas deportistas y el aspecto físico de la Educación Física. Las consecuencias derivadas de estas actitudes son varias ya que las adolescentes no solo pierden una parte vital de su educación y experiencia, sino que además esta pérdida se traduce en términos de perjuicio de aspectos beneficiosos de participación en el deporte tales como salud, auto-estima y diversión (Health Education Authority, 1998). Estas pérdidas no solo ejercen sus efectos en la etapa escolar, sino que se extienden a los estilos de vida posteriores al colegio, así como a los aprendizajes adquiridos a lo largo de la vida (Deem y Gilroy, 1998; Wright y Dewar, 1997).

Estudios realizados en la década de los 90 (García Ferrando, 1993; Mendiara, 1986; Sánchez Bañuelos, 1994; Torre, 1997), han manifestado que las clases de Educación Física, para la mayoría de escolares, resultaban poco satisfactorias y poco divertidas y no suponían un elemento que indujera o motivara hacia la práctica de la actividad física en tiempo de ocio y recreación, así como que la influencia que el profesor de Educación Física ejercía a la hora de motivar al alumnado para que se interesara por el deporte era poco significativa. Por el contrario, según recientes estudios (Casimiro, 1999; Cervelló y Santos-Rosa, 2000; Moreno y Hellín, 2006; Moreno, Rodríguez y Gutiérrez, 2003), la opinión de los alumnos ha cambiado, de tal manera que ahora encuentran que la asignatura en cuestión les gusta más, le dan mayor importancia, está mejor valorada entre los alumnos de Primaria que entre los de Secundaria y, concretamente, más entre los chicos que entre las chicas. Al respecto, una particularidad positiva encontrada en el estudio de Treanor, Graber, Housner y Wiegand (1998) fue que al $84.5 \%$ de los estudiantes le gustaba la Educación Física. A pesar de este hallazgo positivo, se encontraron diferencias significativas en relación al género del discente, de tal manera que, los chicos mostraron un mayor gusto por la asignatura en Educación Secundaria a diferencia de las alumnas, quienes exhibieron un descenso paulatino del gusto o interés por la Educación Física conforme aumentaban en edad, descenso traspasable al nivel de práctica físico-deportiva general, claramente inferior en el caso del sexo femenino (Douthitt, 1994; Hellín, 2003; Jaffee y Richer, 1993; Owen y Bauman, 1992). A este respecto, para Busser, Hyams y Carruthers (1996), Gili-Planas y Ferrer-Pérez (1994) y Lee, Fredenburg, Belcher y Cleveland (1999), las mujeres son más sedentarias porque no les gusta la competición y buscan objetivos a largo plazo que, al ser más difíciles de obtener, dificultaría el mantenimiento de la práctica de ejercicio, mientras que los hombres, al disfrutar del ejercicio por el hecho de realizar una competición, recibirían un refuerzo inmediato que mantendría su conducta. 
Por otro lado, algunos profesionales han señalado la importancia que tiene el comportamiento del profesor en la creación de actitudes positivas hacia la Educación Física en la adolescencia (Dauer y Pangrazi, 1989; Harrinson y Blakemore, 1989), ya que, aspectos como el interés, la satisfacción, el comportamiento de disciplina y el grado de implicación del alumno en la clase, son variables mediatizadas por la percepción que el alumno tiene de la metodología utilizada por el profesor, encontrando que aquellas metodologías que favorecen la autonomía y la toma de decisiones por parte del alumno proporcionan mayor satisfacción, interés, e incluso mayores niveles de práctica física extraescolar (Aicinema, 1991; Chen, 2001; Schemp, Cheffers y Zaichkowsky, 1983; Treasure y Roberts, 2001). En relación con el género del profesor, según Moreno, Sánchez, Rodríguez, Prieto y Mula (2002a, b), aquellos alumnos que tienen un educador de género masculino manifiestan una mayor predilección hacia las clases de Educación Física, otorgándoles éstos, a su vez, mayor importancia a la Educación Física, cuando perciben que su educador tiene un carácter democrático, comprensivo, bueno, alegre, justo, agradable y acepta la iniciativa individual de los alumnos.

\section{Práctica físico-deportiva}

Diferentes investigaciones han mostrado que el sexo femenino participa menos en las clases de Educación Física (Chepyator-Thomson y Ennis, 1997) y practica menos deporte fuera de la etapa escolar, existiendo un incremento del número de adolescentes y mujeres que abandonan la participación en actividades físicas-deportivas (Douthitt, 1994 y Jaffee y Richer, 1993) en comparación con el sexo masculino.

El nivel de participación en actividades físicas y deportivas puede clasificarse atendiendo a tres niveles, deporte y ejercicio físico cotidiano, deporte escolar y deporte de competición. Atendiendo a la práctica deportiva cotidiana, según García Ferrando (2005), en la actualidad, la práctica de los hombres se ha estabilizado en un $41 \%$, mientras que en las mujeres ha experimentado un significativo avance y ha subido hasta el 26\%. Por tramos de edad, se ha observado, también, un incremento de práctica física entre personas con edades comprendidas entre los 25 y 44 años, así como entre las de más de 65 años. En lo que respecta a la práctica deportiva universitaria, según Paris (1996) y Pavón (2004), un 25\% de las estudiantes practican deporte, frente a un 75\% de los chicos. Además, tan solo el $8 \%$ de las alumnas universitarias practican deporte federado frente al $20.7 \%$ de los alumnos masculinos. Según los últimos datos recogidos por García Ferrando (2005), la población prefiere practicar actividad físico-deportiva por su cuenta (68\%), sin preocuparse por la competición $(70 \%)$ y con un grupo de amigos (51\%). Por otro lado, la práctica deportiva de la mujer en el deporte de competición resulta inferior respecto a la del hombre, siendo menor a medida que se eleva la exigencia deportiva. Esta diferencia no es solo cuantitativa sino sobre todo cualitativa, ya que existen diferencias dependiendo de aspectos tales como el interés por el tipo de deporte, expectativas y motivaciones diferentes para la práctica deportiva, facilidad o dificultad de acceso, disposición y distribución del tiempo libre (Sicilia, 
2002; Vázquez, 2001; Vázquez, Fernández y Ferro, 2000) y sobre todo, diferentes razones para comenzar y abandonar la práctica deportiva según el sexo del practicante.

Entre las razones para elegir un deporte, según Garcés de los Fayos (1995) y Vázquez (1989), se encuentran mejorar las habilidades, entretenerse, aprender nuevos movimientos y estar bien físicamente, para ambos sexos. Mientras que García Ferrando (1998, 2001, 2005) señala hacer ejercicio físico, diversión y pasar el tiempo, el gusto por el deporte, mantener y/o mejorar la salud y encontrarse con los amigos, como principales motivos. En el ámbito universitario, el interés para participar en actividades físicas viene motivado por razones vinculadas con el mantenimiento de la forma física y los beneficios ligados a la salud. En concreto, para las alumnas, los motivos más valorados son aquellos relacionados con la salud, liberar la energía acumulada y la imagen personal, mientras que para los alumnos, son las relaciones sociales, el gusto por superarse y la competición (Pavón, 2004). Por su parte, Derry (2002), afirma que mientras los chicos tan solo se interesan en jugar por jugar, las chicas se muestran más interesadas en aprender la manera correcta de jugar, lo que implica el desarrollo de habilidades y estrategias de juego. En este sentido, los juegos de género constituyen un importante rol en las actitudes hacia la Educación Física. Algunos estudios se han centrado en comparar las actitudes de los chicos y las chicas, indicando mayores actitudes de los chicos hacia actividades estimulantes, de riesgo y competitivas, mientras que las chicas mostraron actitudes más favorables hacia actividades físicas que enfatizaban la estética, mejoran la salud y las relaciones sociales (Birtwistle y Brodie, 1991; Blasco, Capdevila, Pintanel, Valiente y Cruz, 1996; García Ferrando, 1998; Gill y Williams, 1996; Hellín, 2003; Hicks, Wiggins, Crist y Moode, 2001).

Entre los deportes más practicados encontramos (García Ferrando, 2005): natación, fútbol, ciclismo, gimnasia de mantenimiento en centro deportivo y senderismo, montañismo o excursionismo, entre otros. Atendiendo a las diferencias por género, las mujeres (Vázquez, 1993; Pavón, 2004) señalan, por orden de mayor participación, la natación, gimnasia, baloncesto, tenis y "bicicleta”, mientras que en mujeres menores de 20 años (Vázquez, 1993) el orden corresponde a: baloncesto, gimnasia, natación, atletismo y tenis. Por el contrario, en los hombres las preferencias son, fútbol, baloncesto, natación, "bicicleta” y tenis. En esta misma investigación, la totalidad de mujeres, sin diferenciación por edad, señalan que los deportes menos apropiados para ellas son el fútbol, boxeo y rugby, coincidiendo con la opinión de los hombres.

Como podemos observar, la mayoría de estudios coinciden en señalar la gimnasia como deporte masivamente practicado por el sexo femenino, diferenciándola claramente del sexo masculino. Por el contrario, la práctica masiva del hombre se centra en el fútbol, deporte que conlleva fuertes connotaciones sociales, caracterizado por ser absolutamente masculino (Clifton y Gill, 1994; Csizma, Witting y Schurr, 1988; Moreno, Rodríguez y Gutiérrez, 1996, 2003; Soto, Soto, Archilla y Franco, 1998).

En lo que respecta a la práctica deportiva escolar, Williams y Woodhouse (1996) mostraron que las chicas presentaban mayores índices de insatisfacción con cinco de las siete actividades desarrolladas en clase de Educación Física. Solo en la categoría de baile, los chicos se encontraban más a disgusto que las chicas. Resultados similares han sido encontrados por Earl y Stennett (1987), determinando que las chicas se mostraban 
más a disgusto con tanta competición, así como por Dickenson y Sparkes (1988) quienes encontraron que las chicas de 11 años preferían la educación saludable y mostraban menor interés por los juegos que los chicos.

Por lo tanto, la actividad física y deportiva que practican las chicas adolescentes y mujeres jóvenes tanto fuera del colegio como en la etapa post-educacional responden a actividades de tipo individual y estéticas tales como el aeróbic y la natación, opuestas estas a los juegos deportivos competitivos tales como el baloncesto y el hockey. En algunos de los casos la oferta de actividades físico-deportivas en las clases de Educación Física no refleja estas necesidades (Sports Council 1998; Williams y Woodhouse, 1996), aspecto que representa desigualdad de oportunidades y discriminación de las chicas y mujeres jóvenes (Scraton, 1992; Talbot, 1986; Williams y Woodhouse, 1996). Wright y Dewar (1997) en su investigación sobre actividad física de mujeres adultas indicaron que éstas experimentan placer en diferentes tipos de actividades, muy pocas de las cuales se encuentran asociadas a actividades tradicionales enseñadas en el colegio.

En lo que respecta a la intensidad de práctica realizada, existen diferencias de género respecto a la práctica en actividades deportivas intensas así como nos muestran diferentes investigaciones (Bauman, Owen y Rushworth, 1990; Leslie, Owen y Sallis, 1999; Leslie, Cerin, Gore, George, Bauman y Owen, 2004; Leslie, Fotheringham, Owen y cols., 2001) que revelan que los hombres se involucran más en deportes intensos que las mujeres en cualquier rango de edad. Es por ello que, si la participación en actividades deportivas en edades tempranas predice dicha práctica en un futuro (Booth, Bauman y Owen, 1997), los bajos índices de participación en actividades físicas intensas encontradas en mujeres, y particularmente en edades por debajo de los 25 años, deberían ser considerados como una prioridad pública hacia la salud (Leslie y cols., 2004).

En cuanto a las razones para el abandono, las mujeres, a pesar de superar los diversos obstáculos que encuentran para practicar actividad físico-deportiva de forma habitual, tanto a nivel competitivo como recreativo, presentan un problema esencial referente al abandono prematuro de la práctica de la actividad. Aunque no existen razones comunes, tanto hombres como mujeres aducen falta de tiempo, no les gusta, por la edad, por pereza, por acabar cansado del trabajo o estudio, por el nivel de exigencia académico, etc. (García Ferrando, 1998, 2001; Pavón, 2004). Según el último trabajo realizado por García Ferrando (2005), las razones más citadas corresponden a salir cansado del trabajo, por pereza y desgana, por la salud, por la edad o por lesiones, entre otras. Por su parte, según Cervelló (2000), existe un paralelismo entre los motivos de práctica físicodeportiva y los motivos de abandono de la misma, de tal manera que, la falta de competencia, el conflicto de intereses, los problemas de tipo grupal y la falta de diversión suelen ser los motivos más citados en el caso del abandono deportivo. En cuanto a los motivos claramente femeninos, influenciados por la sociedad, situamos, miedo a entrenar al anochecer, ayudas domésticas durante los fines de semana, falta de confianza de los padres, etc. Por el contrario, los motivos masculinos hacen referencia a las malas experiencias anteriores y a la pérdida de interés en el deporte (Pavón, 2004). 
Uno de los aspectos a destacar es que las diferencias que se establecen entre los alumnos de diferente género aparecen progresivamente, no por la concepción del deporte que presentan los jóvenes, sino por la de los adultos inmersos en el entorno del sujeto, quienes, con el paso del tiempo, modifican sus actitudes hacia conceptos menos igualatorios, influyendo éstos en la propia concepción del discente. En este sentido, el planteamiento igualatorio de los niños se pierde ligeramente en la adolescencia por la influencia de los "otros significativos" (padres, profesores, iguales, amigos, etc.), por lo que, la importancia de las instituciones y de la familia resulta crucial para conseguir que la tendencia positiva inicial se mantenga o, por el contrario, se dirija a planteamientos ancestrales de actividades propiamente masculinas y/o femeninas.

\section{Importancia de la motivación y la diversión}

En relación con lo indicado anteriormente, los ambientes creados dentro de las clases de Educación Física resultan críticos para el desarrollo de sentimientos tanto positivos como negativos en las adolescentes hacia la actividad física (Derry, 2002), ya que los alumnos que experimentan o perciben sensaciones negativas durante la relación con los iguales en ambientes de actividad física, pueden desarrollar ansiedad o sentimientos negativos que les conducirán a ser sujetos no participantes (Kunesh, Hasbrook y Lewthwaite, 1992).

En este sentido, las investigaciones coinciden en indicar que el aprendizaje de las experiencias positivas relacionadas con la actividad física durante la adolescencia afecta a los niveles de participación de los adultos (Sallis y McKenzie, 1991; Scraton, 1987; The Wellesley College Center for Research on Women, 1992; Wright y Dewar, 1997). Thomas (1985) encontró que uno de los factores contribuyentes a la ausencia de compromiso en el deporte y en el ejercicio hace referencia a malos recuerdos en clase de Educación Física, fundamentalmente, los que hacen referencia a una auto-imagen carente de competencia atlética. Por ello, según Sallis y McKenzie (1991) y The Wellesley College Center for Research on Women (1992), incrementando las experiencias y la participación continuada en actividades físicas durante la edad adolescente, se contribuirá a incrementar los niveles de participación en actividades físicas durante la edad adulta.

En esta línea, según Brustad (1993), Jaffee y Manzer (1992) y Jaffee y Ricker (1993), los factores que afectan a la continuidad de participación en actividad físicas de las chicas (sexo que experimenta menor índice de participación respecto al masculino) incluyen el tiempo dedicado al aprendizaje de habilidades, el nivel de disfrute en la participación, la auto-estima, los beneficios para la salud recibidos a través de la participación y la percepción de competencia atlética. En definitiva, las variables correspondientes a diversión, preocupación por sí mismo y competencia física percibida han sido citadas en la bibliografía especializada como importantes determinantes para la continuidad de actividad física en la edad adulta (Brustad, 1993; Csikszentmihalyi, 1996; Jaffee y Manzer, 1992; Jaffee y Ricker, 1993).

De todas estas variables, el nivel de diversión en la actividad resulta especialmente importante para las chicas debido a la relación existente entre dicha diversión y la 
participación en actividades físicas. Según Florence (1991) y Torre (1997), el alumno motivado se manifiesta en el grupo de clase por diferentes conductas, de tal manera que, se agota, atiende a las explicaciones y las cuestiona, ayuda a los compañeros, quiere hacer más, está alegre, se interesa por su actuación, repite sus ejercicios y se entrena fuera del curso, es decir, siente placer y deseo por ejercitarse y aprender en la clase de Educación Física. Por consiguiente, si las alumnas disfrutan de sus experiencias en las clases de Educación Física, presentarán mayores posibilidades de continuar dichas clases en la etapa universitaria, así como mayores posibilidades de participar en actividades físicas y deportivas en la edad adulta (Sallis, Simons-Morton, Stone, Corbin, Epstein, Faucette, Iannotti, Killen, Klesges, Petray, Rowland y Taylor, 1992). En este sentido, según Carreiro da Costa, Pereira, Diniz y Peirón (1997) y Cervelló (1999), los alumnos poco motivados en Educación Física, se muestran poco satisfechos con las tareas y perciben su competencia inferior a la media.

Según estudios sobre actitudes realizados por Earl y Stennett (1987), Luke y Sinclair (1991), Fernández García (1995) y Hellín y Moreno (2001a,b), entre otros, el grado de satisfacción hacia la Educación Física se encuentra condicionado por factores tales como el contenido del currículum, la práctica deportiva del alumno y de su familia, el género del profesor y del alumno, la fuerte socialización del fenómeno físico-deportivo favorecida por los medios de comunicación y, fundamentalmente, la importancia que los alumnos le conceden a la Educación Física dependiendo de las características del profesor.

Del mismo modo, las investigaciones muestran que uno de los factores que más influye en las experiencias del aprendizaje, y consecuentemente, en la diversión experimentada hacia la actividad física (Gilroy, 1989; Theberge, 1991), hace referencia al autoconcepto (Bropy, 1998, Trew, Scully, Kremer y Ogle, 1999). La auto-imagen de un alumno y consecuentemente la auto-estima afectan tanto a su implicación en el aprendizaje como a los consecuentes niveles de rendimiento (Woodhouse, 1997). Las investigaciones además demuestran diferencias significativas de género de tal manera que, los chicos tienden a tener una visión más positiva de la auto-estima y de su competencia atlética respecto a las chicas (Kremer, Trew y Ogle, 1997; Van Wersch, 1997). Esta habilidad percibida y consecuentemente el auto-concepto puede variar en función de la actividad. En este sentido, el auto-concepto de las chicas, y así su motivación y aprendizaje, estará influenciado por la actividad en cuestión (Woodhouse, 1997).

Los trabajos que analizan las relaciones entre el auto-concepto físico y el género, nos muestran que los chicos y chicas suelen diferir en sus percepciones globales y específicas (Eklund, Whitehead y Welk, 1997). En este sentido, no debemos olvidar la influencia que ejerce el contexto social y cultural sobre las percepciones que configuran los dominios del auto-concepto. De tal manera que, las chicas suelen obtener puntuaciones más bajas en los dominios del auto-concepto físico (Boyd y Hrycaiko, 1997; Goñi y Zulaika, 2000; Hagger, Biddle y Wang, 2005; Moreno y Cervelló, 2005). Estas percepciones desfavorables se obtienen en competencia deportiva, condición física y fuerza (Asçi, Eklund, Whitehead, Kirazki y Koka, 2005; Maïano, Ninot y Bilard, 2004; Welk y Eklund, 2005), atractivo corporal (Hagborg, 1994; Maïano y cols., 2004) y en auto-valoración física (Asçi y cols., 2005; Gutiérrez, Moreno y Sicilia, 1999; 
Maïano y cols., 2004; Welk y Eklund, 2005). En cambio, los resultados obtenidos por Moreno, Cervelló y Vera (2006) en un trabajo con estudiantes de 10, 11 y 12 años, muestran que las chicas obtienen mayores auto-percepciones en apariencia física y fuerza, en tanto la competencia percibida se mantiene más alta en chicos que en chicas.

Esta habilidad percibida y, consecuentemente, el auto-concepto, la habilidad propia de los alumnos ha sido considerada como uno los factores que afecta, esta vez de forma negativa a las actitudes de las chicas hacia la Educación Física (Gilroy, 1989; Theberge, 1991). Resulta ampliamente reconocido que las chicas son más propensas a tener una baja percepción de la habilidad en clase de Educación Física que los chicos (Fox, 1994; Mason, 1995; Shropshire y Carroll, 1998; Trew y cols., 1999). Earl y Stennett (1987) encontraron al respecto que las chicas se avergüenzan más de su bajo nivel de habilidad, siendo ésta una de las razones por la que no les gusta la asignatura. Asimismo, Milosevic (1986) demostró la carencia de confianza de las chicas en su habilidad física.

Es evidente, por tanto, que las chicas sienten que su habilidad resulta restringida en ciertas habilidades, derivando todo ello en una restricción también de su confianza y diversión hacia las mismas (Bandura, 1990; Clifton y Gill, 1994; Lirgg, 1991), particularmente cuando los estereotipos referentes a la habilidad en función del género se encuentran reforzados por la actitud y el comportamiento de algunos profesores (Evans, Davies y Penney, 1996; Pratt, 1985; Scraton, 1992; Wright, 1991).

Tal rol conflictivo puede contribuir a experiencias negativas de las chicas debido a los estereotipos masculinos asociados a muchos deportes y actividades físicas deportivas (Blinde y Greendorfer, 1992; Clifton y Gill, 1994; Lirgg, 1991). Los profesores a menudo sin saberlo incluso refuerzan estos estereotipos a través de intentar proporcionar igualdad de género. Por ejemplo, con la instauración de reglas específicas para chicas, con lo que se les infravalora su potencial y se les envía un fuerte mensaje condescendiente a pesar de que la intención del profesor sea animar la integración de las chicas (Knoppers, 1988).

Es evidente que las alumnas necesitan de una especial atención para descubrir y creer en su propia competencia, pudiendo facilitarse a través de la utilización de estrategias de enseñanza centradas en el alumno, tales como “juegos de enseñanza para el entendimiento" y el uso de diferentes tipos que fomenten la auto-mejora del alumno en ambientes de logro en vez de usar estrategias centradas en la comparación entre sujetos (Fox, 1994; Graydon, 1997). Aunque, igualmente importante resulta la necesidad de dotar de una menor competitividad y de una atmósfera dominada por los chicos en las clases de Educación Física, en las cuales, las chicas sean animadas y permitidas al aprendizaje de forma más positiva (Deem, 1987; Griffin, 1989; Stanworth, 1983; Wright, 1997).

Asimismo, necesitan que les enseñen destrezas y competencias, así como ser animadas y ayudadas en sus niveles de rendimiento, permitiéndoles esto ser personas capazmente razonables en los deportes (Graydon, 1997; Sports Council, 1998).

Ahora bien, en las primeras etapas del alumno antes de llegar a la adolescencia es la familia la que influirá en las actitudes, bien positivas, bien negativas, adquiridas por los 
mismos hacia la Educación Física y la práctica físico-deportiva en general. En este sentido, según McPherson, Curtis y Loy (1989) y Moreno y Cervelló (2003), los alumnos cuyos padres practican algún deporte se encuentran más motivados hacia las clases de Educación Física, variando dicha motivación en función del género al que pertenecen, de tal manera que, según Lewko y Ewing (1980), McPherson (1978) y Snyder y Spreitzer (1973) el grado de ánimo e interés que presentan los chicos hacia la actividad físico-deportiva se encuentra condicionado por el comportamiento del padre, mientras que el de las chicas por el de la madre. La diferencia entre chicos y chicas, según Haywood (1993) y Lewko y Ewing (1980), es que éstas requieren mayores niveles de ánimo y estimulación por parte de todos los miembros de la familia que los chicos.

\section{Aceptación social de las mujeres en el deporte}

Como mencionábamos anteriormente, algunas de las actitudes negativas mostradas por el sexo femenino vienen provocadas por la asociación estereotipada entre deporte y masculinidad. Así, existe una amplia documentación referente a la idea de que el deporte, la actividad física y las clases de Educación Física proyectan una imagen masculinizada en la sociedad oriental, asociándose, por lo tanto, a valores masculinos (Scraton, 1987). Por el contrario, las chicas y las mujeres se encuentran socializadas en la cultura de la "feminidad" a través de su obligada modestia, elegancia, no asertividad, incapacidad física y su preocupación por la estética En este sentido, resulta imprescindible construir la identidad de las alumnas en la etapa adolescente, ya que, según Cockerill y Hardy (1987) aquellas alumnas que no muestran su feminidad son consideradas inmaduras o sexualmente anormales. Consecuentemente, algunas adolescentes y mujeres jóvenes tienden a aferrarse al culto de la feminidad y sucumbirse, así, a las reglas o pautas establecidas por una sociedad patriarcal (Griffin, 1982; McRobbie, 1978).

Sin embargo, esta identidad se encuentra plasmada en las ideas que subyacen de la sociedad, donde se reflejan las posibilidades y los papeles que ambos sexos deben desempeñar frente a la vida en general y frente a la actividad física en particular. En este sentido, según Acker (1994), está aceptado socialmente que existen diferencias de carácter biológico (sexo), de tal manera que existen morfotipos diferentes (la disposición osteo-articular, muscular, la composición corporal, etc. del hombre está determinada, de forma natural, hacia la acción; el morfotipo femenino, la menor proporción de músculo frente a la grasa, las características sexuales, etc. las determinan a actitudes sedentarias y las orientan hacia la maternidad), diferentes capacidades físicas (por naturaleza los hombres son más resistentes, rápidos, ágiles y fuertes que las mujeres), distintos ritmos de aprendizaje (la diferencia de caracteres hace que la mujer tenga mayor dificultad para desarrollar y o aprender determinadas conductas motoras necesarias para la práctica deportiva y el desenvolvimiento físico en general) y la pubertad y la menstruación (una explicación muy tradicionalista afirmaba que la actividad física y el deporte, sobre todo a partir de la pubertad, podían producir “daños” en su capacidad para la maternidad, además de que cambiaría su morfología masculinizándola). Igualmente, en la sociedad subyace la idea de que las mujeres son psicológicamente diferentes $\mathrm{y}$ tienen, por tanto, otros intereses, actitudes $\mathrm{y}$ motivaciones. 
En este sentido, un aspecto fundamental de interés y preocupación para las adolescentes y mujeres jóvenes hace referencia a una gran obsesión por la apariencia física, relacionándose esta obsesión con la ideología de "las mujeres como objeto" (Scraton, 1993). Debido a esta "ansiedad psíquica social” (Graydon, 1997), las chicas tienden a tener una pobre auto-imagen, se muestran más infelices con la forma de su cuerpo y aborrecen con mayor frecuencia la actividad física que los chicos (Aldridge, 1998; Hill y Brackenridge, 1989; Mason, 1995; Milosevic, 1986). Uno de los aspectos que influye en la actitud negativa de las adolescentes hacia la Educación Física hace referencia a la vestimenta específica implícita en la práctica de actividad física, ya que algunas adolescentes se muestran reacias a vestir un equipo que expone el cuerpo o parte del mismo, siendo, además, visto comúnmente carente de elegancia y de moda (Aldrige, 1998; Milosevic, 1986; Scraton, 1987; Shilling, 1993).

Por ello, uno de los objetivos que persiguen algunos autores (Cano, 1991; López, 1992) consiste en dotar a las alumnas de control, poder y diversión obtenido todo ello a través del desarrollo del ejercicio físico. De esta manera, las chicas podrán ser más asertivas y aceptarán su propio cuerpo (y el de los demás), incrementando todo ello sus niveles de confianza, diversión y participación en actividades físico-deportivas. Para conseguir este objetivo, según Griffin (1989) y Talbot (1986), entre otros, los profesores deben evitar mostrar actitudes estereotipadas y proporcionar ambientes de igualdad de sexo siempre que sea posible, así como evitar el enfrentamiento entre chicos y chicas en clases mixtas (Campbell y Broker, 1990; Griffin, 1983, Scraton, 1992) y garantizar tiempo y dedicación a las mismas. De tal manera que, demostrando, así, que la personalidad del profesor y sus estilos de enseñanza no siempre generan efectos positivos en el entusiasmo de las chicas hacia la Educación Física (Mason, 1995).

Al igual que ocurre con los profesores, el entusiasmo de las adolescentes por la Educación Física puede verse incrementado debido a la influencia que ejercen la mujeres deportistas de alto nivel, al proporcionarles un modelo positivo a seguir. Sin embargo, este aspecto se ve limitado debido a la escasa existencia de mujeres deportistas de alto nivel, y más aún, debido al escaso interés y valor que se le otorga a la mujer deportista en nuestra actual sociedad. En este aspecto, Milosevic (1986) encontró que al preguntar a las chicas por sus deportistas preferidos tan solo dos mujeres fueron mencionadas frente a los 62 hombres. Se produce, por tanto, una casi total falta de modelos a imitar por las niñas, adolescentes y mujeres de nuestra sociedad, debido en cierta medida, por un gran número de inconvenientes a las que se enfrentan las mujeres con altas capacidades motrices para llegar a la alta competición en comparación con la situación del grupo masculino de igual capacidad (Alfaro, 2004).

La vida deportiva de una persona integrada en la alta competición atraviesa distintas fases, pudiéndose producir en cada una de ellas acciones positivas o negativas que influirán decisivamente no solo en la vida deportiva sino, también en la totalidad de su ciclo vital. Al respecto, Alfaro (2004) señala algunos de los inconvenientes a los que se enfrenta la mujer para alcanzar el deporte de alta competición. En concreto, en la etapa de iniciación y detección destacamos diferentes aspectos que determina y son responsables del inicio de la mujer en el deporte de alta competición: 
- El ámbito escolar, donde las mujeres tienen menores oportunidades de práctica deportiva que los hombres y generan menos expectativas en el profesorado lo que conlleva a una menor atención y un menor nivel de exigencia.

- El ámbito familiar, donde los padres, generalmente, presentan una menor atención a las aficiones deportivas de las hijas que a la de los hijos y, a su vez, el comportamiento de las niñas se relaciona en mayor medida con la madre que, en general, practica menos deporte y presenta un menor interés por el ámbito deportivo en general.

- Las características personales del sujeto, generalmente las niñas y mujeres se implican en el deporte, motivadas por las relaciones de amistad, no por los resultados alcanzados, todo ello junto con la falta de modelos deportivos femeninos en la sociedad, provoca en la mujer una falta de identificación y motivación con el deporte y su práctica.

En lo que respecta al periodo de desarrollo e incorporación al deporte de alto rendimiento, en el caso de las mujeres, los sacrificios que para todo deportista supone la máxima dedicación, se añaden algunas situaciones específicas tales como el rechazo de la familia y amistades al perder su contacto, la incompatibilidad con estudios y vida familiar, las estructuras deportivas que apoyan en menor medida a las deportistas que se inician, la ausencia de modelos con los que identificarse y la mayoría de entrenadores masculinos existentes en las distintas modalidades deportivas.

En el periodo de optimización, la deportista también se encuentra con una situación diferenciada respecto a la de los deportistas masculinos, ya que, las administraciones deportivas conceden mayor atención al deporte masculino, los recursos económicos indirectos (patrocinadores, etc.) son menores que los recibidos por los hombres, incompatibilidad con los roles establecidos para la mujer por la sociedad (hijos, familia, etc.) y coincidencia con la situación laboral.

En la etapa de abandono, la mujer deportista presenta muy pocas oportunidades para incorporarse al ámbito del entrenamiento o la gestión deportiva, la incorporación a la vida familiar y social resulta complicada al estar desvinculada durante bastantes años de ella, en algunos casos, la deportista carece de formación al haber tenido que abandonar sus estudios durante el periodo de competición y aún queda por estudiar el efecto del entrenamiento de alta competición a largo plazo en el organismo femenino.

\section{Conclusiones}

Son numerosos los estudios que han confirmado la importancia de la Educación Física como influenciadora y potenciadora de la práctica físico-deportiva en la etapa adulta de los jóvenes y adolescentes, así como el importante impacto que ejerce en la salud pública (Haywood, 1991) a través del incremento de la actividad física en los jóvenes 
(Sallis y McKenzie, 1991), y del cultivo de actitudes positivas hacia la participación en la actividad física. Sin embargo, contrario a esta afirmación, existe un descenso considerable a lo largo de la etapa adulta de la adherencia a programas de actividad físico-deportiva, así como un marcado incremento del abandono en la participación de este tipo de actividad, fundamentalmente en el sexo femenino. Por ello, preocupados por esta situación, nos hemos planteado como principal objetivo examinar las actitudes hacia la práctica físico-deportiva según el sexo del practicante, con el fin de conocer y comprender los diferentes motivos que conducen, en relación al sexo, a practicar actividades físico-deportivas, consiguiendo con ello, conocer algunas claves para aumentar la adherencia a programas de estas características durante la etapa adulta.

Diferentes investigaciones han determinado que las actitudes de las adolescentes y mujeres hacia la práctica de actividad física y deportiva tanto en la etapa educativa como post-educacional responden a actividades de tipo individual y estéticas tales como el aeróbic y la natación, mientras que el sexo masculino presenta actitudes hacia la práctica deportiva colectiva y competitiva, tales como el fútbol y el baloncesto. En este sentido, un gran número de estudios se han concienciado acerca de las marcadas actitudes negativas que muestran las adolescentes femeninas hacia la actividad física en general y la Educación Física en particular. Según diferentes autores, estas actitudes son atribuidas al hecho de que el sexo femenino se caracteriza por auto-percibirse menos competente y habilidoso y por sentir menos diversión y disfrute. Unido todo ello a la carencia de modelos deportivos competitivos femeninos con los que asemejarse las niñas, adolescentes y mujeres adultas, a la existencia de unos estereotipos de mujer femenina (no vinculada con el deporte) que, fundamentalmente, refuerzan los padres, así como que en algunos casos la oferta de actividades físico-deportivas en las clases de Educación Física no refleja las necesidades de las alumnas. Aspectos que representan desigualdad de oportunidades y discriminación de las adolescentes y mujeres (Scraton, 1992; Talbot, 1986; Williams y Woodhouse, 1996).

A la vista de los estudios revisados parece conveniente que se profundice en el estudio de la influencia de la experiencia escolar en el comportamiento deportivo de los universitarios, que se analicen las diferencias en el nivel de práctica deportiva entre la población masculina y la femenina, que se estudien los intereses hacia la práctica físicodeportiva de los universitarios, los motivos de práctica, así como de abandono y de no práctica y que se reflexione sobre los intereses y las actitudes mostradas por los participantes hacia la práctica físico-deportiva. Con ello, posiblemente, conseguiremos incrementar las experiencias y, consecuentemente, la participación continuada y la adherencia en actividades físicas durante la etapa adulta, así como reducir, retrasar, y en el mejor de los casos, evitar los periodos de abandono de la población, consiguiendo así, aumentar los indudables beneficios asociados con la práctica físico-deportiva continuada. 


\section{Referencias}

Acker, S. (1994). Género y educación. Reflexiones sociológicas sobre mujeres, enseñanza y feminismo. Madrid: Narcea.

Aldridge, M. (1998). On display and vulnerable: An investigation of the importance and significance of body imafe and sef-image for girls in Physical Education. Tesis Doctoral. Universidad de Southampton.

Alfaro, E. (2004). El talento psicomotor y las mujeres en el deporte de alta competición. Revista de Educación, 335, 127-151.

Aicinema, S. (1991). The teacher and student sttiutdes toward physical education. The Physical Educator, 48, 28-32.

Asçi, F., Eklund, R.C., Whitehead, J.R., Kirazci, S. and Koca, C. (2005). Use of the CY-PSPP in other cultures: a preliminary investigation of its factorial validity for Turkish children and youth. Psychology of Sport and Exercise, 6, 33-50.

Bandura, A. (1990). Perceived self-efficacy in the exercise of personal agency. Journal of Applied Sport Psychology, 2, 128-163.

Bauman, A., Owen, N. and Rusthworth, L. (1990). Recent trends and sociodemographic determinants of exercise participation in Australia. Comm Health Stud, 14, 19-26.

Blasco, T., Capdevila, LI., Pintanel, M., Valiente, L. y Cruz, J. (1996). Evolución de los patrones de actividad física en estudiantes universitarios. Revista de Psicología del Deporte, 9, 51-63.

Blinde, E.M. and Greendorfer, S.L. (1992). Conflict and college sport experiences of women athletes. Women in Sport and Physical Activity J ournal, 1, 97-113.

Birtwistle, G.E. and Brodie, D.A. (1991).Children's attitudes toward activity and perceptions of physical education. Health Education Research, 6, 465-478.

Booth, M.L., Bauman, A., Owen, N. and cols. (1997). Physical activity preferences, preferred sources of assistance and perceived barriers to increased activity among physically-inactive Australians. Preventive Medicine, 26, 131-137.

Boyd, K.R. and Hrycaiko, D.W. (1997). The effect of a physical activity intervention package on the self-esteem of pre-adolescent and adolescent females. Adolescence, 32, 127, 693-709.

Brophy, J. (1998). Motivating students to learn. Champaign: Human Kinetics.

Brustad, R.J. (1993). Who will go out and play? Parental and psychological influences on children's attraction to physical act.ivity. Pediatric Exercise Science, 5, 210-223.

Busser, J.A., Hyams, A.L. and Carruthers, C.P. (1996). Differences in adolescent activity participation by gender, grade and ethnicity. Journal of Park and Recreation Administration, 14, 1-20. 
Campbell, A. and Brooker, N. (1990). Tom, Dick and/or Harriet: Some interventionist strategis against boys' sexist behaviors. En E. Tutchell (Ed.), Dolls and Dungarees (pp. 45-67). Milton Keynes, Open University Press.

Cano, S. (1991). Alternativas para conseguir una Educación Física no sexista. En Actas de las I Jornadas para docentes de Educación Física de la Región de Murcia. Murcia.

Carlson, T.B. (1995). We hate gym: Student alienation from physical education. Journal of Teaching in Physical Education, 4, 467-477.

Carreiro Da Costa, F., Pereira, P., Diniz, J. and Peirón, M. (1997). Motivation, perception de competence et engangement moteur des eleves dans des classes d'education physique. Revue de l'Education Physique, 37, 83-91.

Casimiro, A.J. (1999). Comparación, evolución y relación de hábitos saludables y nivel de condición física-salud en escolares, entre final de Educación Primaria (12 años) y final de Educación Secundaria Obligatoria (16 años). Tesis Doctoral. Granada.

Cervelló, E. (1999). Variables psicológicas relacionadas con la elección de tareas con diferente nivel de dificultad: implicaciones para el desarrollo de programas de entrenamiento psicológico motivacional en deporte. Motricidad, 5, 35-52.

Cervelló, E. (2000). Una explicación de la motivación deportiva y el abandono desde la perspectiva de la Teoría de Metas: Propuestas para favorecer la adherencia a la práctica deportiva. En Actas del I Congreso Hispano-Portugués de Psicología. Santiago de Compostela.

Cervelló, E. y Santos-Rosa, F.J. (2000). Motivación en las clases de Educación Física: Un estudio de la perspectiva de las metas de logro en el contexto educativo. Revista de Psicología del Deporte, 9, 51-70.

Chen, A. (2001). A theoretical conceptualization for motivation reseach in physical education: An integrated perspective. Quest, 53, 35-58.

Chepyator-Thomson, J.R. and Ennis, C.D. (1997). Reproduction and resistance to the culture of feminity and masculinity in secondary school physical education. Research Quarterly for Exercise and Sport, 68, 89-99.

Clifton, R.T. and Gill, D.L. (1994). Gender differences in self-confidence on a feminine typed task. Journal of Sport and Physical Activity Journal, 1, 97-113.

Cockerrill, S.A. and Hardy, C. (1987). The concept of femininity and its implications for physical education. British J ournal of Physical Education, 18, 149-151.

Cothran, D.J. and Ennis, C.D. (1998). Curricula of mutual worth: Comparisons of students' and teachers'curricular goals. Journal of Teaching in Physical Education, 17, 307-326.

Csizma, K.A., Witting, A.F. and Schurr, K.T. (1988). Sport stereotypes and gender. Journal of Sport and Exercise Psychology, 10, 62-74.

Csikszentmihalyi, M. (1996). Creavity. New York: Harper Collins.

Dauer, V. and Pangrazi, R. (1989). Dynamic physical education for elementary school children. New York: Macmillán Publishing Company.

Deem, R. (1987). Unleisured lives: Sport in the context of women's leisure. Women's Studies International Forum, 18, 423-432. 
Deem, R. and Gilroy, S. (1998). Physical activity, life-long learning and empowerment: Situating sport in women's leisure. Sport, Education and Society, 3, 89-104.

Derry, J.A. (2002). Single-sex and coeducation physical education: perspective of adolescent girls and female physical education teachers (research). Melpomene Journal, 22, 17-28.

Derry, J.A and Phillips, D.A. (2004). Comparasions of selected student and teahcer variables in all-girls and coeducational Physical Education Environments. Phsycial Education Environments. Physical Educator, 61, 23-34.

Dickerson, B. and Sparkes, A. (1988). Pupil definition of physical education. British J ournal of Physical Education Research Supplement, 2, 6-7.

Dishman, R.K. (1988). Exercise adherence: Its impact on public health. Champaign, IL: Human Kinetics.

Douthitt, V.L. (1994). Psychological determinants for adolescent exercise adherence. Adolescence, 115, 711-722.

Earl, L.M. and Stennet, R.G. (1987). Student attitudes toward physical and helth education in secondary school in Ontario. Canadian Association for Health, Physical Education and Recreation Journal, 53, 4-11.

Eklund, R.C., Whitehead, J.R. and Welk, G.J. (1997). Validity of ghe children and youth physical self-perceptions profile: a Confirmatory Factor analysis. Research Quarterly for Exercise and Sport, 68, 249-256.

Ennis, C.D. (1996). Students' experiences in sport-based physical education: (More than) apologies are necessary. Quest, 48, 453-456.

Erickson, E.H. (1968). I dentidad, juventud y crisis. Madrid:Taurus.

Evans, J., Davies, B. and Penney, D. (1996). Teachers, teaching and the social construction of gender relations. Sport, Education Society, 1, 165-184.

Fernández García, E. (1995). Actividad física y género: Representaciones diferenciadas en el futuro profesorado de Educación Primaria. Tesis Doctoral. Madrid.

Florence, J. (1991). Tareas significativas en Educación Física Escolar. Barcelona: INDE.

Fox, K. (1994). Understanding young people and their decisions about physical activity. British Journal of Physical Education, 25, 15-19.

Garcés de los Fayos, E.J. (1995). La situación actual de la práctica deportiva en el alumnado femenino: actitudes de las niñas y las adolescentes ante el deporte en la Región de Murcia. Murcia: Consejería de Sanidad y Asuntos Sociales de la C.A. de Murcia.

García Ferrando, M. (1993). Tiempo libre y Actividades Deportivas de la Juventud en España. Madrid: Ministerio de Asuntos Sociales.

García Ferrando, M. (1998). Estructura social de la práctica deportiva. En M. García Ferrando, N. Puig y F. Lagardera (Eds.), Sociología del deporte (pp. 41-67). Madrid: Alianza.

García Ferrando, M. (2001). Las prácticas deportivas de la población española en el cambio de siglo. En V. Carratalá, J.F. Guzmán y M.A. Fuster (coord.), Nuevas aportaciones al estudio de la Actividad Física y el Deporte (pp. 95-97). Valencia: Universidad de Valencia. 
García Ferrando, M. (2005). Encuesta sobre hábitos deportivos de los españoles: Avance de resultados. Madrid: Centro de Investigaciones Sociológicas.

Gili-Planas, M. y Ferrer-Pérez, V. (1994). Práctica deportiva y estereotipos de género: un estudio en la Comunidad Autónoma de las Islas Baleares (CAIB). Revista de Psicología de Deporte, 5, 81-88.

Gill, D.L. and Williams, L. (1996). Competitive orientations and motives of adult sport and exercise participants. Journal of Sport Behavior, 19, 307-318.

Gilroy, S. (1989). The embodyment of power: gender and physical activity. Leisure Studies, 8, 163-171.

Goñi, A. and Zulaika, L.M. (2000). Relationships between physical education classes and enhancement of fifth Grade pupils' self-concept. Perceptual and Motor and Skills, 91, 146-150.

Graham, G. (1995). Physical education through students' eyes and in students' voices: Introduction. J ournal of Teaching in Physical Education, 14, 364-371.

Graydon, J. (1997). Self-confidence and selg-esteem in physical education and sport. En G. Clarke and B. Humberstone (Eds.), Researching women and sport (pp. 67-89). London: Macmillan.

Griffin, C. (1982). Cultures of feminity: romance revisited. Centre for Contemporary Cultural Studies Occasional Paper, Women series, 69.

Griffin, P.S. (1983). "Gymnastics, is a girl's thing": Student participation and interaction patterns in a middle school gymnastics unit. In T. Templin and J. Olson (Eds.), Teaching in physical education (pp. 71-85). Champaing, IL: Human Kinetics.

Griffin, P.S. (1989). Gender as a socializing agent in Physical Education. En T.J. Templin and P.G. Schempp (Eds.), Learning to teach: Socialization into Physical Education (pp. 219-233). Indianapolis, IN: Benchmark Press.

Gutiérrez, M., Moreno, J. A. y Sicilia, A. (1999). Auto-concepto físico y práctica deportiva de una muestra de estudiantes universitarios. Comunicación presentada al IV Congrés de les Ciències de l'Esport, l'Educació Física i la Recreació. Lleida, INEFC.

Hagbor, W.J. (1994). The Rosenberg self-esteem scale and Harter's self-perception profile for adolescents: a concurrent validity study. Psychology in the Schools, 30, 132-136.

Hagger, M., Biddle, S. and Wang, C.K. (2005). Physical Self-concept in adolescence: Generalizability of a multidimensional, hierarchical model across gender and grade. Educational and Psychology Measurement, 65, 297-322.

Harrinson, J. and Blakemore, C. (1989). Instructional strategies for secondary school physical eduation. Dubuque, IA: William C. Brown Publishers.

Haywood, K. (1991). The role of physical education in the development of active lifestiles. Research Quality for Exercise and Sport, 62, 151-156.

Haywood, K. (1993). Life Span Motor Development. Canadá: Human Kinetics Publishers.

Health Education Authority (1998). Promoting physical activity with young women: guidelines. London: Health Education Authority. 
Hellín, P. (2003). Hábitos físico-deportivos en la Región de Murcia: implicaciones para la elaboración del currículum en el ciclo formativo de Actividades FísicoDeportivas. Tesis Doctoral. Murcia.

Hellín, P. y Moreno, J.A. (2001a). Actitudes hacia la Educación Física de los alumnos de ciclos formativos. En Actas del II Congreso de Ciencias de la Actividad Física y el Deporte (pp. 549-557). Valencia, Universidad de Valencia.

Hellín, P. y Moreno, J.A. (2001b). Importancia de la Educación Física. En Actas del II Congreso Internacional de Educación Física y Diversidad (pp. 693-708). Murcia, Conserjería de Educación y Universidades.

Hicks, M.K., Wiggins, M.S., Crist, R.W. and Moode, F.M. (2001). Sex differences in grade three students' attitudes toward physical activity. Perceptual and Motor Skills, 93, 97-102.

Hill, J. and Brackenridge, A.C. (1989). My body's a complete wreck: the contribution of physical education to physical confidence. Physical Education Review, 12, 147-157.

Jaffee, L. and Manzer, R. (1992). Girl's perspective: Physical activity and selfesteem. Melpomen. A J ournal for Women's Health Research, 11, 14-23.

Jaffee, L. and Ricker, S. (1993). Physical activity and self-esteem in girls: The teen years. Melpomene. A J ournal for Women's Health Research, 12, 19-26.

Jones, D.A., Ainsworth, B.E., Croft, J.B., Macera, C.A., Lloyd, E.E. and Yusuf, H.R. (1998). Moderate leisure time physical activity: Who is meeting the public health recommensations? A national cross-sectional study. Archives of Family Medicine, 7, 285-289.

Knoppers, A. (1988). Equity for excellence in Physical Education. Journal of Physical Education, Recreation and Dance, 59, 54-58.

Kremer, J., Trew, K. and Ogle, S. (1997). Young people's involvement in sport. London: Routledge.

Kunesh, M.A., Hasbrook, C.A. and Lewthwaite, R. (1992). Phsyical activity socialization: Peer interactions and affective responses among a sample of sixth grade girls. Sociology of Sport Journal, 9, 385-396.

Lee, A.M. (1997). Contribution of research on student thinking in physical education. Journal of Teaching in Physical Education, 16, 262-277.

Lee, A.M., Fredenburg, K., Belcher, D. and Cleveland, N. (1999). Gender differences in clhildren's conceptions of competence and motivation in physical education. Sport, Education and Society, 4, 161-174.

Leslie, E., Cerin, E., Gore, C.J., George, A., Bauman, A. and Owen, N. (2004). Gender, age and educational-attainment differences in australian adults' participation in vigorous sporting and fitness activities. Journal of Physical Activity and Health, 1, 377-388.

Leslie, E., Fotheringham, M.J., Owen, N. and cols. (2001). Age-related differences in physical activity levels of young adults. Medicine \& Science in Sports \& Exercise, 33, 255-258.

Leslie, E., Owen, N. and Sallis, J. (1999). Inactive Australian college student's preferred activities, sources of assistance and motivators. American Journal of Health Promotion, 13, 197-199.

Lewko, J. and Ewing, M. (1980). Sex differences and parental influences in sport involvement of children. Journal of Sport Psychology, 2, 62-68. 
Lirgg, C.D. (1991). Gender differences in self-confidence in physical activity: a meta-analysis. Journal of Sport and Exercise Psychology, 13, 294-310.

López, C. (1992). Hacia un planteamiento coeducativo de la Educación Física. En Actas de las IV J ornadas Internacionales de Coeducación. Valencia.

Luke, M.D. and Sinclair, G.D. (1991). Gender differences in adolescents' attitudes toward Physical Education. J ournal of Teaching in Physical Education, 11, 31-46.

Maïano, C., Ninot, G. and Bilard, J. (2004). Age and gender effects on global selesteem and physical self-perception in adolescents. European Physical Education Review, 10, 53-69.

Mason, V. (1995). Young people and sport in England, 1994. Londond: Sports Council.

McPherson, B. (1978). Children in sport: A contemporary antohology. Canadá: Champaign: Human Kinetics Books.

McPherson, B., Curtis, J. and Loy, J. (1989). The social significance of sport. Canadá, Champaign: Human Kinetics Books.

McRobbie, A. (1978). Working class girls and the culture of femininity. En Centre for Contemporary Cultural Studies (Ed.), Women take issues. London: Hutchinson.

Mendiara, N. (1986). Análisis de la Educación Física en el sistema educativo español: Pasado, presente y futuro. En Actas del Seminario Mujer y Deporte. Madrid: INEF.

Milosevic, L. (1986). Fairplay. Gender and physical education. Leeds City: Council Department of Education.

Moreno, J.A. y Cervelló, E. (2003). Pensamiento del alumno hacia la Educación Física: Su relación con la práctica deportiva y el carácter del educador. Enseñanza, 21, 345-362.

Moreno, J.A. y Cervelló, E. (2005). Physical self-perception in spanish adolescentes: gender and involvement in physical activity effects. Journal of Human Movement Studies, 48, 291-311.

Moreno, J.A., Cervelló, E. y Vera, J.A. (2006). Evaluación participativa y responsabilidad en educación física. Manuscrito sin publicar.

Moreno, J.A. y Hellín, G. (2006). ¿Interesa la Educación Física al alumnado de educación secundaria obligatoria? Manuscrito en revisión.

Moreno, J.A., Rodríguez, P.L. y Gutiérrez, M. (1996). Actitudes hacia la Educación Física: elaboración de un instrumento de medida. En Actas del III Congreso Nacional de Educación Física de Facultades de Educación y XIV de Escuelas Universitarias de Magisterio (pp. 507-516). Guadalajara, Universidad de Alcalá.

Moreno, J.A., Rodríguez, P.L. y Gutiérrez, M. (2003). Intereses y actitudes hacia la Educación Física. Revista Española de Educación Física, 9, 14-28.

Moreno, J.A., Sánchez, M., Rodríguez, D., Prieto, M.P. y Mula, C. (2002a). ¿Puede el comportamiento del profesor influir en la valoración que el alumno realiza de la Educación Física?. En A. Díaz, J.A. Moreno y P.L. Rodríguez (Eds.), III Congreso de Educación Física e Interculturalidad. Murcia: Conserjería de Educación.

Moreno, J.A., Sánchez, M., Rodríguez, D., Prieto, M.P. y Mula, C. (2002b). Valoración de la Educación Física según la importancia concedida por el alumno. 
En A. Díaz, J.A. Moreno y P.L. Rodríguez (Eds.), III Congreso de Educación Física e Interculturalidad. Murcia: Conserjería de Educación.

Owen, N. and Bauman, A. (1992). The descriptive epidemiology of physical inactivity in adult Australians. International Journal of Epidemiology, 21, 305310.

Paris, F. (1996). El deporte en las universidades españolas. Análisis de la encuesta sobre su organización, práctica y equipamientos. Serie I.C.D. Madrid: Consejo Superior de Deportes y Ministerio de Educación y Cultura.

Pavón, A. (2004). Motivaciones e intereses de los universitarios murcianos hacia la práctica físico-deportiva. Tesis Doctoral. Murcia: Universidad de Murcia.

Portman, P. (1995). Who is having fun in physical education classes? Experiences of sixth-grade students in elementary and middle schools. Journal of Teaching in Physical Education, 44, 445-553.

Pratt, J. (1985). The attitudes of teachers. En J. White, R. Deem, L. Kant and M. Cruickshank (Eds.), Girl friendly schooling (pp. 79-90). London: Methuen.

Sallis, J.F. and McKenzie, T.L. (1991). Physical education's role in public health. Research Quaterly far Exercise and Sport, 62, 124-137.

Sallis, J.F., Simons-Morton, B.G., Stone, E.J., Corbin, C.B., Epstein, L.H., Faucette, N., I annotti, R.J., Killen, J.D., Klesges, R.C., Petray, C.K., Rowland, T.W. and Taylor, W.C. (1992). Determinants of physical activity and interventions in youth. Medicine and Science in Sports and Exercise, 24, 248-257.

Sánchez-Bañuelos, F. (1994). Seminario Permanente de Investigación sobre Didáctica de la Educación Física. Madrid: INEF.

Schempp, P.G., Cheffers, J. and Zaichkowsky, L.D. (1983). Influence of decisionmaking on attitudes, creativity, motor skills and self concept in elementary children. Research Quarterly for Exercise and Sport, 54, 183-189.

Scraton, S. (1987). Boys muscle in where angels fear to tread. Girls' subcultures and physical activities. En J. Horne, D. Jary and A. Tomlinson (Eds.), Sport, Leisure and Social Relations. London: Routledge and Kegan Paul.

Scraton, S. (1992). Shaping up to womanhood: gender and girls' physical education. Milton Keynes: Open University Press.

Scraton, S. (1993). Equality, coeducation and physical education in secondary schooling. En J. Evans (Ed.), Equality, Education and Physical Education (pp. 139-153). London: The Falmer Press.

Shilling, C. (1993). The body, class and social inequalities. En J. Evans (Ed.), Equality, education and physical education (pp. 35-49). London: Falmer, Press.

Shropshire, J. and Carrol, B. (1997). Family variables and children's physical activity: influence of parental exercise and socio-economic status. Sport, Education and Society, 2, 95-116.

Shropshire, J., Carrol, B. and Yim, S. (1997). Primary school children's attitudes to physical eduation: gender differences. European Journal of Physical Education, 2, 23-38.

Sicilia, A. (2002). La investigación sobre el pensamiento del alumnado. Una revisión desde la Educación Física. Revista de Educación, 331, 577-613.

Snyder, E. and Spreitzer, E. (1973). Family influence and involvement in sports. Research Quarterly for Exercise and Sport, 44, 249-255. 
Soto, A., Soto, E., Archilla, I. y Franco, C. (1998). La condición del género en las preferencias por la actividad física y deportiva. En A. García, F. Ruiz y A.J. Casimiro (Eds.), Actas del II Congreso Internacional sobre la Enseñanza de la Educación Física y el Deporte Escolar (pp. 131-135). Málaga: Instituto Andaluz del Deporte.

Sports Council (1998). Women and sport: Policy and frameworks for actions. London: Sports Council.

Stanworth, M. (1983). Gender and schooling. London: Hutchinson.

Talbot, M. (1986). Gender and physical education. British Journal of Physical Education, 4, 120-122.

The Wellesley College Ccenter for Research on Women. (1992). The AAUW Report: How Schools Shortchange Girls. NEA: Professional Library.

Theberge, N. (1991). Reflection son the body in the sociology of sport. Quest, 43, 123-134.

Thomas, J.R. (1985). Remembrance of things past and best forgotten. Physical Education Review, 8, 7-13.

Torre, E. (1997). La actividad físico-deportiva extraescolar y su interrelación con el área de Educación Física en el alumnado de Enseñanzas Medias. Tesis Doctoral. Granada: Universidad de Granada.

Treanor, L., Graber, K., Housner, L. and Wiegand, R. (1998). Middle school student's perceptions of coeducational and same-sex Physical Education classes. J ournal of Teaching Physical Education, 18, 43-56.

Treasure, D.C. and Roberts, G.C. (2001). Students'perceptions of the motivational climate, achievement beliefs, and satisfaction in physical education. Research Quarterly of Exercise and Sport, 72, 165-175.

Trew, K., Scully, D., Kremer, J. and Ogle, S. (1999). Sport, leisure, and perceived self-competence among male and female adolescent. European Physical Education Review, 5, 53-73.

Van Wersch, A. (1997). Individual difference and intrinsic motivations for sport participations. En J. Kremer, R. Trew and S. Ogle (Eds.), Young people's involvement in sport (pp. 57-78). London, Routledge.

Vázquez, B. (1989). La Educación Física en la Educación Básica. Madrid: Gymnos.

Vázquez, B. (1993). Actitudes y prácticas deportivas en las mujeres españolas. Madrid: Ministerios de Asuntos Sociales.

Vázquez, B. (2001). La cultura física y las diferencias de género en el umbral del siglo XXI. En J. Devís (Ed.), La educación física, el deporte y la salud en el siglo XXI (pp. 213-226). Alcoy: Marfil.

Vázquez, B., Fernández, E. y Ferro, S. (2000). Educación Física y Género: Modelo para la observación y el análisis del comportamiento del alumnado y del profesorado. Madrid: Gymnos.

Welk, G.J . and Eklund, B. (2005). Validation of the children and youth physical selfperceptions profile for young children. Psychology of Sport and Exercise, 6, 5165.

Williams, V. and Woodhouse, J. (1996). Delivering the discourse, Urban adolescents' perceptions of physical education. Sport, Education and Society, 1, 201-213. 
Woodhouse, J. (1997). So, how do the pupils feel?. The Bulletin of Physical Education, 32, 40-45.

Wright, J. (1991). I say what I mean, said Alice: An analysis of gendered discourse inphysical education. J ournal of Teaching in Physical Education, 10, 210-225.

Wright, J. (1997). The construction of gendered context in single sex and coeducationl physical education lesson. Sport, Education and Society, 2, 1.

Wright, J. and Dewar, A. (1997). On pleasure and pain: Women speak out about physical activity. En G. Clarke and B. Humberstone (Eds.), Researching women and sport (pp. 111-134). London: Macmillan. 